\title{
Sistemas Emocionales y la Tradición Evolucionaria en Psicología
}

\section{Emotional systems and the evolutionary tradition in psychology}

\author{
Carlos E. Zerpa ${ }^{1}$ \\ Departamento de Ciencia y Tecnología del Comportamiento \\ Universidad Simón Bolívar, Caracas Venezuela
}

(Recepción: Mayo 2009 - Aceptación: Julio 2009)

\begin{abstract}
Resumen
Se presenta una breve revisión de la tradición evolucionaria en Psicología de los sistemas emocionales. Se describe la aproximación de Darwin al estudio de la expresión emocional en humanos, conforme a los principios derivados de la teoría de la evolución por selección natural. Posteriormente se exponen las posturas teóricas de los representantes actuales más destacados en Psicología de las emociones, desde la perspectiva evolucionaria. Finalmente, se presentan algunas de las tendencias actuales y líneas de investigación futura sobre el tema y algunas conclusiones referidas a la importancia del estudio de las emociones para la Psicología actual.

Palabras clave: Emoción, Psicología Evolucionaria, Teoría de la Evolución.

Abstract

A brief review paper is presented about evolutionary tradition in emotional Psychology systems. Darwin approximation view to study of expression of emotion in humans is described. Some actual theoretical perspectives and researchers and future tendencies in emotional systems research since Darwinian tradition in Psychology and implications for disciplinary field are discussed.

Key words: Emotion, Evolutionary Psychology, Evolutionary theory.
\end{abstract}

1 Correspondencia a: Carlos E. Zerpa, Departamento de Ciencia y Tecnología del Comportamiento, Universidad Simón Bolívar, Venezuela. E-mail: czerpa@usb.ve 
Introducción: el estudio de las emociones en Psicología.

Si en la historia de la Psicología contemporánea han existido temas difíciles de estudiar, sin duda que el de las emociones ocupa un lugar privilegiado en dicha clasificación. En efecto, abordar lo que hoy conocemos como "sistemas emocionales" representó durante muchos años un reto para la Psicología científica, sobre todo porque entre 1940 y mediados de los años de 1970 el conductismo era la fuerza que predominaba en la Psicología experimental y las necesidades de una verdadera ciencia psicológica excluían la investigación de la emoción en tanto se consideraba un fenómeno privado e inobservable. De esta manera se prefirió restringir la consideración del tema a conceptos como "respuesta emocional condicionada” desviándose así su estudio esencial y dándole más bien primacía al análisis del condicionamiento como explicación de la conducta. Lo interesante del caso es que a mediados de la década de 1970 este panorama cambia progresivamente para dar paso a un renovado interés sobre la emoción como fenómeno psicológico desde diferentes perspectivas: cognitiva, social y recientemente, evolucionaria.

Desde la perspectiva evolucionaria, heredera actual del legado de Charles Darwin (1809-1882) y su teoría de la evolución de las especies ("El origen de las especies” Darwin, 1859/1959), el estudio de las emociones se inició con una obra no muy conocida de Darwin, al menos para la comunidad de psicólogos en Latinoamérica, titulada "La expresión de las emociones en hombres y animales" (Darwin, 1872/1965) en la cual su autor analizó el rol de la evolución en la forma como los humanos expresan sus emociones básicas y la función que actualmente cumplen para la especie. En otras palabras, la perspectiva evolucionaria de la Psicología en el estudio de las emociones da cuenta tanto de las características propias de su expresión así como de su valor adaptativo. Desde el legado de Darwin se trata entonces de una interpretación actual a través de una panorámica de la estructura covariante del pasado evolutivo de la humanidad. ¿Cuán similares son las expresiones emocionales en personas y animales superiores? ¿Cómo se explica esta similaridad? ¿Qué tan universal es la similaridad de la expresión emocional en la especie humana? En esencia, responder a estas interrogantes es el objetivo fundamental del presente trabajo al abordar el estudio de las emociones básicas desde la perspectiva darwiniana.

Lo que se expone seguidamente se ha organizado en cinco (5) partes: a saber, una descripción de lo que puede entenderse por emociones básicas; la explicación de la perspectiva darwiniana del estudio de las emociones enfatizando sus antecedentes; cuestiones de método y principios subyacentes; un análisis de los aspectos que actualmente se consideran de importancia en el estudio de las emociones básicas desde la Psicología evolucionaría contemporánea; y, finalmente, se incluyen algunas conclusiones.

\section{Emociones básicas}

Las emociones están implicadas en el afrontamiento de diferentes tareas fundamentales de la vida cotidiana; de allí su valor adaptativo cuando se relacionan a episodios de pérdidas, frustraciones, etc. El adjetivo de "básicas" enfatiza el rol que la evolución ha jugado en la expresión tanto única como común de las mismas, así como de la función que actualmente cumplen para la especie humana (Ekman, 1994).

En tal sentido, un criterio fundamental para clasificar una emoción como básica es su carácter universal, común a la especie humana (Kalat y Shiota, 2006). Según tal criterio, una emoción básica puede ocurrir en todas las sociedades (con independencia del asunto cultural, quiere decirse) exceptuando aquellas personas que genética o morfológicamente o fisiológicamente hayan nacido con alguna alteración que modifique o impida la expresión "típica” de estados como felicidad, tristeza y miedo, entre otros.

La Psicología evolucionaria ha asumido el estudio de seis (6) expresiones emocionales que se consideran de carácter universal (es decir, se observan en la mayoría de las culturas del mundo); el cuadro 1 da cuenta de ellas. 
Cuadro 1. Seis expresiones emocionales universales que son objeto de estudio de la Psicología evolucionaria actual.
1. Sorpresa
2. Tristeza
3. Miedo
4. Disgusto-repugnancia-asco
5. Felicidad
6. Ira-enojo-cólera

Para Kalat y Shiota (2006) las emociones básicas facilitan una respuesta funcional a un evento específico o prototípico de la vida cotidiana y aparecen en etapas muy tempranas del desarrollo evolutivo. Las características de las emociones básicas (en tanto [e]- mociones: movimiento) incluyen la peculiaridad de que ocurren en presencia de un evento externo que las elicita y una duración normalmente breve, aún cuando el estímulo persista (o bien, desaparezca).

El interés en el tema de las emociones ha motivado a muchos investigadores a proponer importantes teorías en un intento por explicar cómo funcionan las cosas en el mundo emocional. Las diversas teorías de la emoción organizan el conocimiento sobre dicho concepto haciendo referencia a aquellos aspectos no observables que se vinculan con ella. En el caso particular de la teoría de la evolución por selección natural de Darwin (1859/1959) puede decirse que permite la organización de un vasto cuerpo de conocimientos acerca de las características anatómicas de animales diversos extintos que aún viven (Cornelius, 1996); también permite la formulación de predicciones acerca de posibles cambios en la morfología de las especies. Este carácter continuo de la evolución de las características de los organismos vivos es lo que ha permitido abordar las emociones (básicas) conforme al punto de vista evolucionario en Psicología. En efecto, Ekman (1999) e Izard (2009) afirman que las emociones no pueden ser comprendidas sin atender a consideraciones de su historia evolutiva y la contribución de las mismas a la supervivencia del individuo y su especie.

\section{La perspectiva darwiniana}

\section{a. Antecedentes}

Hacia el año de 1839 y en ocasión del nacimiento de William Darwin, primogénito del insigne investigador inglés, emerge el interés de Charles Darwin en la observación de la expresión de las emociones, bajo el supuesto teórico de la existencia de un continuo en la naturaleza (desde los animales hasta los humanos) que resulta en evidencia de cómo opera la selección natural sobre el comportamiento. El volumen 7 de la revista "Mind" de Julio de 1877 publica un artículo de Darwin titulado "bosquejo biográfico de un infante" donde describe buena parte de las observaciones que sirvieron previamente para la organización de su libro de 1872 ("la expresiones de emociones en hombres y animales”). Cornelius (1996) refiere que estos textos darwinianos constituyen una mezcla tanto de observaciones rigurosas y teorizaciones como de antropomorfismo criticable en la actualidad.

En última instancia, las formulaciones de Darwin en estos documentos ponen de relieve su interés en establecer un paralelismo entre la conducta de los humanos y la de los animales. Pero este punto de vista, vale la pena aclarar, tiene como partida la noción evolutiva plasmada en la teoría de Jean Baptiste Lamarck, expuesta por vez primera en un texto de 1800 llamado "el sistema de los animales sin vértebras", teoría en la que Lamarck postula su doctrina transformista, una aproximación pre-darwiniana de gran importancia en la carrera por la teoría evolucionista. 
Otro antecedente importante del punto de vista evolutivo en el estudio de las emociones lo aportaron los trabajos de Charles Bell (1774-1842), un cirujano inglés que afirmó que los músculos que son utilizados para la expresión de las emociones fueron creados exclusivamente para tales fines únicamente en humanos, postura que entró en franco desacuerdo con las ideas de Darwin, quien se encargó a lo largo de su obra de refutarla: la teoría de Bell se oponía a la de Darwin en cuanto a que este último planteaba la existencia de un continuo en la expresión emocional ubicando en un extremo a los animales y en el otro a los humanos. Para Darwin, el reto era probar que los mismos principios que explican la expresión de las emociones en los animales también podían aplicarse a la explicación de la expresión de las emociones en los humanos.

Según Darwin, (1872/1965) la expresión emocional consiste en acciones que son directa o indirectamente útiles bajo ciertos estados de la mente, para aliviar o gratificar ciertas sensaciones; cuando se induce el mismo estado de la mente, aunque sea de forma débil, hay una tendencia a través de la fuerza del hábito para que el mismo movimiento sea nuevamente ejecutado.

En otras palabras, esta teoría afirma que la expresión emocional sirve a un propósito específico en la especie y permite satisfacer cierta clase de demandas que se le hacen al organismo, lo cual se traduce en la idea de que la expresión de las emociones realmente no se desarrolló para expresar emociones, tal como lo entendemos en la cotidianidad, sino para otros fines. La vinculación con la evolución viene dada por el lado de la finalidad adaptativa a la que sirve la satisfacción de tales demandas. En la historia evolutiva entonces los organismos que tuvieron mayor éxito adaptativo al emplear la expresión emocional para fines de supervivencia lograron transmitir sus genes a su descendencia. El punto de vista evolucionario en Psicología estudia los registros que existen de estos procesos biológico-fisiológicos para realizar sus actuales formulaciones.

\section{b. Elementos de la teoría darwiniana de las emociones}

La explicación de las emociones en cuanto a su expresión la resolvió Darwin con el planteamiento de lo que se conoce como "principios generales de expresión"; tales planteamientos reflejarían la acción de la selección natural sobre las emociones. Se exponen seguidamente, siguiendo a ChólizMontañez (1995):

-Principio de hábitos útiles asociados: los hábitos o movimientos que son de utilidad para satisfacer deseos o disminuir sensaciones, por ejemplo, se hacen tan recurrentes que terminan manifestándose en situaciones que no requieren de dicho patrón de comportamiento como respuesta. Expresiones de emociones particulares que han tenido importancia adaptativa al resultar útiles en una clase particular de contexto pueden manifestarse en situaciones completamente diferentes debido a su valor evolutivo (aunque en algún sentido esto puede realmente oponerse al principio de selección natural de la teoría de la evolución de las especies).

-Principio de Antítesis. Se producen respuestas motoras contrarias cuando para un hábito consolidado en la especie se genera un estado de ánimo contrario al que originalmente elicita dicho hábito; esto ocurre a pesar de que las respuestas motoras contrarias al hábito puedan resultar poco útiles en la situación. Para Darwin, la relación entre una expresión y su opuesta es esencialmente mecánica y ocurre solo por asociación.

-Principio de acción directa del sistema nervioso. Durante un episodio de gran excitación para el organismo, la fuerza nerviosa asociada a la misma puede dar pie a movimientos expresivos. El sistema nervioso central puede someterse a factores que elicitan alta actividad en él (lo que se denomina “excitación”) liberándose fuerzas nerviosas (de acuerdo a Darwin, pueden expandirse en sensaciones intensas, pensamientos activos, movimientos violentos o incremento en la actividad de las glándulas endocrinas).

Ejemplos de cada uno de estos principios los encontramos en las siguientes situaciones, ilustradas por el mismo Darwin: 1) puede observarse un comportamiento típico en un gato que está sometido a una situación de peligro como lo es la aproximación de un perro; la postura que adopta (la columna arqueada, la cola baja casi entre las patas traseras y el pelo erizado) puede también 
observarse en cualquier otra situación que represente una amenaza para el animal. Esto lo explica el primer principio (hábitos útiles asociados); 2) considerando esta vez el comportamiento de algunos perros, es posible verlos de pronto persiguiendo motocicletas en movimiento, con gran agitación y fiereza y en un momento inmediato posterior, se encuentran completamente dóciles. La situación es explicable por el segundo principio (o principio de antítesis); 3) considerando finalmente un hecho fortuito como tropezar con un objeto y golpearse con fuerza una mano; el comportamiento asociado inmediato puede ser, por ejemplo, agitar rápidamente la mano en un intento por "liberar" el dolor; hecho explicable por el tercer principio (o de acción directa del sistema nervioso).

En el planteamiento darwiniano, se asume que los patrones de respuesta-expresión de emociones son determinados genéticamente en su forma pero el aprendizaje por asociación puede jugar un importante papel al modificar la expresión misma y su manifestación en unas situaciones y no en otras. Siendo innatas las emociones básicas y heredadas de los antepasados de la especie, muestran la continuidad filogenética en términos de su expresión y la evidente relación entre la fisiología y el comportamiento que existe en la obra de Darwin.

Por ejemplo, en el caso del llanto, asociado a la tristeza como emoción básica, la aproximación darwiniana afirma que las lágrimas tienen como función la protección de los ojos cuando se emiten gritos de forma violenta. Fisiológicamente ocurre un congestionamiento de los vasos sanguíneos de los ojos y en consecuencia, la contracción de los músculos alrededor del globo ocular les protege. Igualmente, el aumento de la presión estimula la secreción de las glándulas lacrimales y se producen las lágrimas. De acuerdo a Cornelius (1996) las lágrimas cumplirían una función de protección de los ojos bajo determinadas circunstancias. Más allá de eso, las lágrimas no representarían realmente una expresión de sufrimiento sino que cumplirían un papel diferente al cuidar los ojos durante un episodio particular de tensión.

En consecuencia, el llanto, si se considera como expresión del sufrimiento humano, lo es en tanto en la historia de la especie se ha asociado con acciones como gritar y agitación que aumentan la presión sobre los globos oculares; esto indica que el significado social que se le asigna al llanto ha sido dado solo por casualidad; esto vale también para otras emociones básicas que accidentalmente han sido asociadas a determinadas cualidades expresivas.

\section{El método de investigación de Darwin: características y críticas}

Sobre cuestiones de método, el de Darwin tuvo sus matices. Posiblemente el interés científico de este investigador, ya muy conocido en la década de 1870, le motivó a buscar evidencias sólidas de sus hipótesis evolucionistas referidas a la expresión emocional, más allá de sus propias observaciones sistemáticas y su dedicación a la investigación documental sobre el tema. Para comprobar su teoría, ideó un procedimiento de recolección de datos que tenía dos variantes: 1) la administración de cuestionarios; y 2) el análisis de fotografías. Es necesario recordar que en la época de Darwin, la fotografía era un recurso escaso y muy costoso.

Con respecto al primer procedimiento, básicamente se trataba de la recopilación de registros anecdóticos de las observaciones que hacían terceros en diferentes partes del mundo. En efecto, enviaba un cuestionario tipo (que solo tenía 16 ítemes de preguntas abiertas) a misioneros que se encontraban de servicio en el exterior (de Inglaterra), ubicados en muy diversos contextos socioculturales y luego los analizaba buscando los elementos de interés para su investigación. De acuerdo a Cornelius (1996), el número de instrumentos que fundamentaron sus hipótesis no superaron los 36 ejemplares y con base a ellos logró comparar al europeo de su época con personas de otras culturas de menor desarrollo. En efecto, las comparaciones realizadas le permitieron encontrar similitudes entre unas y otras personas, independientemente de la cultura de origen.

Similarmente, con respecto al segundo procedimiento, el análisis de fotografías le llevó a seleccionar un banco de imágenes que mostraban expresiones emocionales que de acuerdo a su criterio (e inspirado en los trabajos de Duchene de Bolougne de la década anterior, 1860) reflejaban similaridades interculturales. Luego realizó ensayos en los que mostraba la selección de fotografías 
a diferentes personas para que juzgaran el tipo de emoción que se expresaba en cada una. Igualmente concluye en la independencia de la expresión emocional con respecto a la cultura.

Un par de observaciones a los procedimientos anteriores pueden mencionarse: 1) la muestra de datos que recolectó en principio parece pequeña y es difícil decir que garantizaba aspectos esenciales de la observación de la conducta como las nociones de confiabilidad o validez; 2) utilizó un criterio personal acerca de lo que constituía una expresión emocional representativa de las categorías de conducta sobre las que se interesó (las emociones básicas). Pero aún así, las ideas de Darwin encontraban cierta evidencia empírica en sus trabajos de recolección de datos; la similaridad intercultural en la expresión emocional reflejaba la herencia evolutiva que vincula estrechamente a la especie humana con sus parientes primates. Alineado con su teoría de la selección natural, la expresión de las emociones, concluía, probablemente evolucionó debido a que conferían algún tipo de ventaja reproductiva o para la supervivencia en los individuos que las mostraban, incrementando así las probabilidades para vivir.

\section{Paul Ekman y Carroll Izard: la tradición evolucionaria darwiniana en la Psicología actual de las emociones.}

Los psicólogos evolucionarios afirman que los ancestros de la especie humana enfrentaron una multitud de problemas adaptativos como evadir depredadores, obtener alimentos, hallar refugio, atraer parejas y comunicarse con semejantes, entre muchos otros, los cuales requirieron una única solución; de allí que planteen que la mente humana consiste de una gran variedad de mecanismos psicológicos distintivos que han derivado de cambios adaptativos específicos. En tal sentido es razonable pensar que en los humanos han evolucionado emociones distintas, cada una diseñada para enfrentar una serie específica de problemas adaptativos (Haselton y Ketelaar, 2005).

De acuerdo a Cornelius (1996) la postura actual de la Psicología evolucionaria con respecto a la expresión emocional parte de una hipótesis común de referencia, la cual formula que si la expresión emocional está sujeta a la evolución por selección natural, entonces los miembros de una misma especie deben exhibir las mismas expresiones emocionales. La investigación asociada ha mostrado fuerte evidencia para apoyar la idea de que algunas expresiones emocionales son universales en la especie humana. Tales emociones universales son las que se categorizaron como básicas y que se muestran en el cuadro 1.

La investigación actual de las emociones conforme a los presupuestos evolucionarios en Psicología es liderada por Paul Ekman, de la Universidad de California y Carroll Izard, de la Universidad de Delaware. Cada uno ha desarrollado tanto programas de investigación sobre la expresión emocional universal, como teorías explicativas de esta variable.

En el caso de Ekman (1999), defiende la idea de que existe un número finito de emociones básicas que son separables de otras en función de sus atributos característicos. Para el caso de las emociones que considera son negativas (miedo, ira, disgusto, tristeza y desprecio) difieren en su apreciación, eventos que le anteceden, respuesta conductual probable y elementos de fisiología asociados. En el caso de las emociones que considera positivas (amor y felicidad) ocurre lo mismo. Kalat y Shiota (2006) describen para cada caso (desde la misma perspectiva evolucionaria y considerando también las emociones positivas como la alegría y otras como la sorpresa) los componentes fisiológicos que la literatura refiere hallarse en la investigación sobre el tema. Por ejemplo, la biología de la tristeza incluye la activación del sistema nervioso autónomo como actividad fisiológica asociada a la producción de lágrimas y al hecho de gritar (puede entenderse, lamentos de tristeza). También se reportan incrementos en la concentración de cortisona en sangre, lo cual igualmente se observa en la expresión del miedo así como la activación de la amígdala. Para otras emociones básicas se consiguen también indicadores particulares. No obstante, Ekman afirma que el significado del término "básico" en las emociones puede también vincularse a una segunda acepción de corte constructivista-social debido a que en el transcurso de la evolución el aprendizaje en las diferentes culturas ha podido moldear precisamente la forma en que ellas se expresan. De allí su interés en establecer ciertos atributos, a la manera de criterios clasificatorios, para la diferenciación de emociones básicas y de 
otras emociones que probablemente se han construido en el mundo social. Las características que distinguen a las emociones básicas de otras se pueden organizar en las categorías que se exponen en el cuadro 2.

Aún así, la perspectiva de Ekman de la expresión emocional se articula a la noción de valor adaptativo conforme permite enfrentar y cumplir con tareas fundamentales para la vida. Es decir, cada emoción básica prepara al individuo en una dirección la cual, en el curso de la evolución, ha resultado mejor que otras respuestas o soluciones a las demandas del ambiente en circunstancias relevantes para el logro de metas (enfrentar peligros inmediatos, afrontar pérdidas irreparables, entre otras).

Cuadro 2. Categorías de diferencias que distinguen a las emociones básicas de otras (Ekman, 1999).

Señales distintivas universales.

Fisiología distintiva.

Activación automática, en sintonía con el evento elicitante.

Distinción universal en eventos antecedentes.

Distintiva apariencia en el desarrollo.

Presencia en otros primates.

Rápido inicio.

Corta o breve duración.

Distintividad en pensamientos y en imágenes de memoria.

Distintiva experiencia subjetiva.

Algunos de los procedimientos de Ekman en sus investigaciones han incluido el estudio intercultural de las expresiones faciales (Ekman y Friesen, 1971). La investigación realizada con participantes que no habían tenido un importante contacto con la cultura occidental se diseñó para presentarles cierto número de fotografías que mostraban a niños o adultos manifestando expresiones faciales de alguna emoción de categoría básica. Previamente los reactivos habían sido evaluados como pertinentes y categorizados para cada emoción según criterio de expertos. Los hallazgos reportados muestran que el $70 \%$ de las personas participantes podían juzgar y categorizar correctamente cada una de las fotografías de emociones que se les presentaron. Estos resultados coinciden en un alto grado con lo que los mismos investigadores reportan para el caso de personas que pertenecen a la cultura occidental. Si bien, únicamente la expresión emocional del miedo fue en cierta medida confundida con la expresión de sorpresa, la mayoría de las personas de uno y otro grupo reconocen o identifican correctamente cada expresión emocional, independientemente de la cultura y de si se trataba de personas letradas o no-letradas.

Este tipo de estudios, en suma, permitió a Ekman y su equipo concluir que para cierto número de emociones su reconocimiento podía darse de forma universal, con independencia de la cultura. Concluye así que la socialización no podía ser un mecanismo apropiado para explicar estos hallazgos y alinea sus trabajos conforme a la tradición darwiniana-evolucionista. En efecto, Ekman cree que la función de la emoción moviliza al organismo para enfrentar rápidamente importantes encuentros interpersonales, preparándole para realizar aquel tipo de actividades que han sido adaptativas en el pasado. El pasado se refiere a lo que en su historia ha sido útil para la adaptación exitosa del individuo o su especie al entorno. 
La perspectiva de Ekman dio paso para el análisis de aquellas situaciones que, por influencia del aprendizaje social, moderaban la expresión de ciertas emociones. Hay situaciones sociales en las que resulta más apropiada la expresión de una emoción y no de otra. Esto evidencia el hecho de que las personas son capaces de suprimir o alterar la expresión de la forma como se siente en función del contexto en el que se encuentre. Cornelius (1996) y Workman y Reader (2004) se refieren a esta supresión con el término de "reglas del despliegue" y se corresponde a la forma como Ekman definió el papel del aprendizaje en el enmascaramiento de la expresión facial. Se trata de patrones aprendidos en el proceso de socialización dentro del contexto de una cultura particular que condicionan el que una persona o grupo de personas manifieste una emoción en una situación dada, modificando su expresión de acuerdo a lo apropiado o no del momento. Algunas diferencias entre la expresión facial de personas anglosajonas y japonesas pueden ilustrarlo. El duelo en Japón es un acontecimiento que se expresa de forma menos dramática que en América. Un norteamericano en Japón, sometido a una pérdida dolorosa que implique duelo (la muerte de un pariente cercano, por ejemplo) posiblemente muestre una expresión de tristeza más discreta, ajustada al patrón cultural japonés, que si se encontrara en su país. La regla del despliegue en este caso invita al personaje a enmascarar la expresión de lo que siente y ajustarse a lo que el medio social en el que se encuentra le exige. Para el japonés, la regla del despliegue es precisamente la discreción.

Por otro lado, los trabajos de Carroll Izard sobre las emociones en la Universidad de Delaware también han tenido como punto de partida el legado evolucionista darwiniano. Para Izard (2009) las emociones derivan del desarrollo evolutivo y neurobiológico y han jugado un eminente papel adaptativo para la especie humana. Este investigador se ha dedicado a la elaboración de la teoría diferencial de las emociones (TDE) y focaliza sobre el concepto de "sentimientos emocionales". Propone que los sentimientos emocionales son realmente una fase (mas no una consecuencia) de la actividad neurobiológica o de la expresión corporal de la emoción. Esta postura puede estar en contraste con perspectivas evolucionarias asociadas como la de Damasio (1999), quien afirma que la respuesta del cerebro constituye la emoción o la expresión corporal de la emoción distinguiendo el "sentimiento de emoción” como una consecuencia de la expresión neurobiológica.

Desde el darwinismo neural se articula a la idea de que las estructuras permanecen sobre la base de sus implicaciones en los mecanismos de selección natural. Por ejemplo, los cambios en la estructura cerebral. Estos cambios juegan un papel crítico para cada individuo y para la especie en general. El trabajo de la evolución aporta registros que son analizables para explicar como emergen las emociones. No obstante, para Izard los sentimientos emocionales si bien pueden activarse y ser influenciados por procesos perceptuales, conceptuales y otros procesos no cognitivos, no pueden ser creados por ellos. Conforme a las afirmaciones de otros evolucionistas (por ejemplo, Edelman y Tononi, 2000), el desarrollo de estas tempranas cualidades ocurre en extensos periodos sobre la base de discriminaciones multimodales centradas en lo corporal y cargadas por los sistemas propioceptivo, kinestésico y el sistema nervioso autónomo que ya están presentes en el embrión humano y en el cerebro del infante. En consecuencia, una emoción concreta no puede ser creada, aprendida o enseñada vía procesos cognitivos.

De acuerdo con Cornelius (1996), los trabajos de Izard refieren que las emociones fundamentales tienen entonces una inherente función adaptativa. Los elementos que se le pueden atribuir están vinculados a: 1) un sustrato neural específico innatamente determinado; 2) un patrón característico de expresión facial o neuro-muscular; 3) una cualidad distintiva subjetiva o fenomenológica. Sin embargo, con respecto al segundo atributo, la teoría actual del autor supone que la expresión facial puede ser o no parte de la actividad neurobiológica crítica involucrada en el sentimiento emocional.

En cuanto a las emociones que identifica, concluye en la existencia de diez (10) emociones fundamentales: 1) interés-excitación; 2) júbilo; 3) sorpresa; 4) pena/dolor-congoja/angustia; 5) repugnancia/asco; 6) desprecio; 7) miedo; 8) vergüenza; 9) ira/cólera; 10) culpa. Se retoma en este punto la afirmación de Izard (2009) que refiere que una emoción es una fase de la actividad neurobiológica que es sentida por el organismo, inclusive si se prescindiera de la corteza cerebral 
y que puede variar en cuanto a su nivel de intensidad. Un atributo importante de la teoría es que postula un gradiente para los niveles de sensación de la emoción, como ocurre con otras actividades de orden neurobiológico. Estos sentimientos van desde un nivel de intensidad bajo, poco perceptible, hasta un nivel alto que puede llegar a ser extremo. Esta cualidad del sentimiento de la emoción en el organismo ha sido de fundamental importancia en el proceso de evolución de la conciencia y en los procesos afectivos, cognitivos y de acción que se vinculan con la conducta orientada a metas. El sentimiento sería la cualidad de los procesos neurobiológicos pero no la actividad neurobiológica per se.

Pero conviene aclarar que la postura actual de Izard distingue (como consecuencia de las formulaciones anteriores) entre dos tipos amplios de emociones: por un lado las emociones básicas (positivas o negativas), referidas al proceso afectivo dinámico generado por la evolución del cerebro primitivo en función de la validez ecológica de la estimulación. Por otro, los esquemas emocionales o interacciones dinámicas emoción-cognición. Las primeras han sido fundamentales para la adaptación, el desarrollo y la supervivencia del individuo manifestándose en la ontogenia y son susceptibles al cambio en su desarrollo. Las segundas se refieren a la emoción en interacción dinámica con procesos perceptuales y cognitivos que influyen sobre la mente y la conducta. Estos esquemas son elicitados por procesos de activación pero también por imágenes, recuerdos y pensamientos y otros procesos de orden más psicofisiológicos y neurobiológicos como el intercambio de neurotransmisores y el cambio periódico en el nivel de las hormonas. Son influenciados por las diferencias individuales, el aprendizaje y el contexto social y cultural.

Sin embargo, el componente de sentimiento de un esquema emocional dado es cualitativamente idéntico al sentimiento de una emoción básica. Pueden tener una duración breve (como las emociones básicas) o bien transcurrir durante un tiempo indefinido, principalmente por la interacción continua con procesos cognitivos que proveen significado para regularlos y utilizarlos. Concluye que los sentimientos emocionales constituyen el sistema motivacional primario para la conducta humana y que son un factor primario en la organización, evolución y las operaciones de la conciencia y los diferentes niveles de auto-conciencia.

\section{Tendencias de la tradición evolucionaria en la Psicología contemporánea de las emociones}

Variadas son las aproximaciones actuales y futuras al tema de las emociones que se derivan de la perspectiva evolucionista darwiniana y que han progresado en los últimos 15 años. Por ejemplo, Randolph Nesse (1990) y Bárbara Frederickson (Frederickson, Cohn, Coffey, Pek y Findel, 2008), en la misma línea de Ekman (1999), han propuesto funciones particulares para estados emocionales particulares. Estados negativos como el miedo y la angustia han podido evolucionar para ubicar a las personas en el estado psicológico y fisiológico correcto para enfrentarse con circunstancias adversas. Estos estados internos son similares a respuestas conductuales apropiadas, que de forma específica denominan "tendencias de acción”. Para Nesse, las emociones positivas como el amor y los componentes de la felicidad han recibido menos atención que las negativas (lo mismo afirman Kalat y Shiota, 2006). A igual que Frederikson et al. (2008), afirma que las emociones positivas pueden ubicar a la persona en la condición apropiada para establecer relaciones sociales satisfactorias (las emociones negativas estrechan el campo de atención; las positivas, "abren la mente”). Más recientemente Nesse se ha dedicado a la explicación evolucionaria de los síntomas de emociones como la depresión en tanto área de estudio de la Psicología clínica y la Psiquiatría (Nesse, 2006).

Ekman (1999) por su parte ha venido indagando en el papel específico de las emociones en la regulación de la manera como las personas piensan, abordando ámbitos vinculados a la memoria, la imaginación y las expectativas, afirmando que la relación entre emoción y pensamiento no es tan solo función del aprendizaje social debido al estrecho vínculo con las características biológicas heredadas por la especie. De esta manera desarrolla líneas de investigación relacionadas con la experiencia subjetiva de la emoción y los sentimientos emocionales sobre la base de la existencia de 15 emociones que considera básicas: diversión, ira-cólera-enojo, desprecio, satisfacción, ascoaversión-repugnancia, vergüenza-pudor, excitación, miedo-temor, culpa, orgullo en la ejecución, consuelo, tristeza-melancolía/pena-dolor, satisfacción, placer sensorial y deshonra-oprobio. La idea 
de Ekman está centrada en la existencia de familias de emociones determinadas por las diferencias individuales y las ocasiones específicas en las cuales pueden ocurrir.

Otras direcciones futuras incluyen líneas de investigación en desarrollo representadas por Morris y Keltner (2000) y Zielemberg y Pieters (2005) quienes desde la perspectiva evolucionaria de las emociones se han introducido en campos como la toma de decisiones emocionales para buscar nuevos usos de la economía experimental, vinculando la teoría de juegos con las emociones. En otro orden, también se tienen importantes avances en la investigación de las emociones como programas cognitivos superordinados que coordinan los pensamientos y las conductas en respuesta a cambios adaptativos específicos. Sin embargo, estas aproximaciones implícitamente no distinguen entre el concepto de emoción y el de cognición, lo cual impide la contrastación entre la emoción y la razón (Haselton y Ketelaar, 2005). Aún así, y de acuerdo con los presupuestos de Izard (2009), la distinción entre regulación de la emoción y utilización de la emoción puede proveer nuevos insights en la comprensión de la independencia e interdependencia de estos dos constructos.

\section{Conclusiones}

El propósito de este trabajo fue presentar una revisión de la perspectiva evolucionaría en Psicología de las emociones. La Psicología evolucionaria de las emociones asume una explícita perspectiva adaptativa bajo la premisa de que la mente es el compromiso de diversas adaptaciones cada una de las cuales es producto de la selección natural y sexual que ha operado en el transcurso de muchas generaciones en la historia de la especie humana (Buss, Haselton, Shackelford, Bleske y Wakefield, 1998).

La Psicología evolucionaria de las emociones ha buscado evidencia para apoyar la idea de la universalidad de la expresión emocional en los humanos. En tal sentido ha centrado sus esfuerzos en tres fuentes de información (Workman y Reader, 2004): 1) la comparación de la expresión de las emociones con otros primates; esto ha sugerido la existencia de un ancestro común; 2) los estudios interculturales, que sugieren que las diversas culturas del planeta reconocen y experiencian las mismas emociones básicas; 3) la identificación de estructuras neurológicas asociadas con estados emocionales específicos; en particular la amígdala se ha observado con intensa actividad en emociones como el miedo; también el área orbito-frontal de la corteza del cerebro parece estar involucrada en la apreciación de indicios emocionales en otras personas. Otras estructuras neurológicas parecen asociarse con diferentes emociones básicas.

Una implicación importante de la perspectiva evolucionaria de las emociones quizás es su potencialidad heurística; la ciencia puede aportar significados y elementos de interés sobre las emociones para la formulación de leyes con base a las regularidades de posible observación. Esto puede suponer, conforme a la posición de Cosmides y Tooby (2000) el descubrimiento de insospechados estados emocionales que caraterizó la conducta de los ancestros de la especie humana; posiblemente, también la perspectiva permita la predicción de los cambios de la expresión emocional en el futuro, conforme al sentido que la teoría de la evolución ha tenido para la ciencia en general y la funcionalidad de la misma. Indudablemente, todo lo anterior muestra la importancia de las emociones en la arquitectura de los procesos adaptativos de la especie humana y el interés determinante de la Psicología en uno de sus pilares conceptuales de mayor relevancia en su historia. 


\section{Referencias}

Buss, D. M.; Haselton, M. G.; Shackelford, T. K.; Bleske, A. L. \& Wakefield, J. (1998). Adaptations, exaptations and spandrels. American Psychologist, 53, 533-548.

Chóliz-Montañez, M. (1995). La expresión de las emociones en la obra de Darwin. En F. Tortosa, C. Civera y C. Calatayud (comps.). Prácticas de historia de la Psicología. Valencia: Promolibro.

Cornelius, R. R. (1996). The science of emotion. Research and tradition in the psychology of emotions. New Jersey: Prentice Hall.

Cosmides, L., \& Tooby, J. (2000). En M. Lewis y J. M. Haviland-Jones (eds.). Handbook of emotion, 2da. Ed. New York: Guilford.

Damasio, A. R. (1999). The feeling of what happens: body and emotion in the making of consciousness. New York: Harcourt Brace.

Darwin, Ch. (1859/1959). El origen de las especies por medio de la selección natural. México: Universidad Nacional Autónoma de México.

Darwin, Ch. (1872/1965). The expression of the emotions y man and animals. Chicago: The University of Chicago Press.

Darwin, Ch. (1877, Julio). A biographical sketch of an infant. Mind, 7, 2, 285-294.

Edelman, G. M. \& Tononi, G. (2000). A universe of consciousness: how matter becomes imagination. New York: Basic Books.

Ekman, P. (1994). All emotions are basic. En P. Ekman y R. J. Davidson (eds.). The nature of emotions: fundamental questions. New York: Oxford University Press.

Ekman, P. (1999). Basic emotions. En T. Dalgleish y M. Power (eds.). Handbook of cognition and emotion. Sussex, U. K.: John Wiley \& Sons.

Ekman, P., \& Friesen, W. V. (1971). Constants across cultures in the face and emotion. Journal of Personality and Social Psychology, 17, 1, 124-129.

Frederickson, B. L., Cohn, M. A., Coffey, K. A., Pek, J., \& Findel, S. M. (2008). Open hearts build lives: positive emotions, induced through bring-kidness mediation, build consequencial personal resources. Journal of Personality and Social Psychology, 95, 5, 1045-1082.

Haselton, M. G. \& Ketelaar, T. (2005). Irrational emotions or emotional wisdom? The evolutionary Psychology of emotions and behavior. En J. Forgas (ed.). Hearts and minds: affectives influences on social cognition and behavior. (Frontiers of social Osychology series). New York: Psychology Press.

Izard, C. (2009). Emotion theory and research: highlights, unaswered questions and emerging issues. Annual Review of Psychology, 60, 1-25.

Kalat, J. W. \& Shiota, M. N. (2006). Emotion. Belmont, CA: Thomson.

Nesse, R. M. (1990). Evolutionary explanations of emotions. Human Nature, 1, 261-289.

Nesse, R. M. (2006). The evolutionary significance of depressive symtoms: different adverse situations lead to different depressive symtoms patterns. Journal of Personality and Social Psychology, 91, 2, 316-330.

Workman, L., \& Reader, W. (2004). Evolutionary psychology: an introduction. Cambridge University Press. Consultado en Enero 21, 2009 de http://books.google.com/books 